\title{
Analyzing the Dual Space of the Saturated Ideal of a Regular Set and the Local Multiplicities of its Zeros
}

\author{
Xialiang $\operatorname{Li}^{1}$ and Wei $\mathrm{Niu}^{2}$ \\ ${ }^{1}$ Dongguan University of Technology - City College \\ ${ }^{2}$ Beihang University
}

March 19, 2021

\begin{abstract}
In this paper, we are concerned with the problem of counting the multiplicities of a zero-dimensional regular set's zeros. We generalize the squarefree decomposition of univariate polynomials to the so-called pseudo squarefree decomposition of multivariate polynomials, and then propose an algorithm for decomposing a regular set into a finite number of simple sets. From the output of this algorithm, the multiplicities of zeros could be directly read out, and the real solution isolation with multiplicity can also be easily produced. As a main theoretical result of this paper, we analyze the structure of dual space of the saturated ideal generated by a simple set as well as a regular set. Experiments with a preliminary implementation show the efficiency of our method.
\end{abstract}

\section{Hosted file}

multsqf.pdf available at https://authorea.com/users/402608/articles/514420-analyzing-thedual-space-of-the-saturated-ideal-of-a-regular-set-and-the-local-multiplicities-of-itszeros 